\title{
Exploration of the economic and quality of life impact on carers of individuals undergoing community rehabilitation
}

\section{Tracy A Comans, Cindy J Sealey, Merehau C Mervin, Ruth N Barker}

Background/Aims: For people with neurological conditions, informal carers form an important part of the rehabilitation journey. The aim of the current study was to assess the economic and quality of life outcomes for someone caring for a person undertaking a community rehabilitation programme. Methods: A prospective study was conducted of a community rehabilitation service with baseline and 3-month follow-up data. Participants in this study were carers of people undergoing rehabilitation. Data was collected on time spent caring and on employment status using a questionnaire. Quality of life was measured using the carer experience scale.

Results: Carers spend an average of 28 hours per week in caring activities and this was similar after rehabilitation was complete. Replacement by a paid worker would cost over $A \$ 650$ a week. Quality of life of the carer did not significantly change after rehabilitation was completed. Thirty-seven percent of carers have ceased work because of their caring duties.

Conclusions: Three months may be too short to measure the impact of rehabilitation on carers. Carers represent a substantial economic resource and carers are at increased risk of poverty because of their caring duties. Integrating carers into the rehabilitation process may help improve outcomes for carers as well as for clients of community rehabilitation services.

Key words: Carers Community rehabilitation Economic evaluation

Submitted: 5 October 2015; accepted following double blind peer review: 29 March 2016 eople who experience a stroke or brain injury are likely to develop physical or cognitive residual disability that will prevent them from functioning independently in the community (Low et al, 1999). They are likely to require rehabilitation and long-term additional support, which is usually provided in the form of informal care by family or close friends (Al-Janabi et al, 2008). Informal carers enable the individual to remain in the community; most importantly, informal carers play a substantial role in the rehabilitation of the individual (Low et al, 1999; Gunnell et al, 2000). In Australia, data from the 2003 Australian Survey of Disability, Ageing and Carers (SDAC) estimated that there were 474600 people who were the primary carers of a person because of disability or age.

The economic costs of caregiving need to be evaluated given the expected increase in unpaid provision of caring, with the rise of the ageing population and associated health conditions (Goranitis et al, 2014). In Australia, substantial economic costs and financial hardship to individuals are incurred as a result of informal caring responsibilities (Hill et al, 2011). Many caregivers report stopping work because of the time spent caring (Hill et al, 2011). In 2009, Australian primary caregivers reported spending upwards of 40 hours a week in caring duties. Of those primary carers, only $27 \%$ were in the workforce (Australian Bureau of Statistics, 2012). The resultant inability to work is highlighted by the $65 \%$ of primary caregivers with incomes in the lowest two quintiles. Understanding the economic impact of caring can assist in setting priorities for government investment in this area.

Caring for individuals with disabilities is also likely to impact on the carer's quality of life. The
Tracy A Comans, Principal research fellow, Menzies Health Institute Queensland, Griffith University, Brisbane, Australia,

Metro North Hospital and Health Service District, Brisbane, Australia; Cindy J Sealey, Research officer, James Cook University, Townsville, Australia, Northern Australia Primary Health Limited, Townsville, Australia; Merehau C Mervin Research fellow, Menzies Health Institute Queensland, Griffith University, Brisbane, Australia;

Ruth N Barker Associate professorRehabilitation, James Cook University, Townsville, Australia, Northern Australia Primary Health Limited, Townsville, Australia.

Correspondence to: Tracy A Comans

Email:

t.comans@griffith.edu.au 
hours spent caring for individuals with disability impacts greatly on a carer's ability to engage in social activities outside the house, without the person they are caring for. Many studies have shown that caregiving tasks often result in a physical and emotional burden due to the numbers of tasks to perform or the time required for each task (Hughes et al, 1999; Chipchase and Lincoln, 2001; Blake et al, 2003). Caregiving tasks also create social problems for the caregiver as carers forego the personal time spent in social, cultural or physical activities away from the home (Iecovich, 2008). Investigation of the full range of impact of caregiving on the carer in terms of financial, economic, social and psychological wellbeing would provide an understanding of the areas that most require service and attention. Understanding such aspects of caring are required to provide clear direction in the development of programmes to support informal carers.

Rehabilitation is expected to improve the functioning of the person and therefore should reduce the need for assistance in tasks such as self-care and meal preparation. The aim of the current study was to measure the impact of community rehabilitation on the economic outcomes and quality of life for carers caring for a person with a neurological condition.

\section{METHODS}

The current study was part of a larger evaluation of the costs and benefits of a community rehabilitation service in Townsville, Queensland in Australia. Ethical approval for the study was obtained through the James Cook University Human Research Ethics Committee. Participants in the main study were people with mainly neurological conditions who were current clients at the community rehabilitation service. They were interviewed at the beginning of their rehabilitation programme (i.e. baseline) and at 3-month follow-up, by which time most had completed their rehabilitation. The main study recruited 206 participants with a mean age of 61 (standard deviation: 15.3) years. Participants' neurological conditions were classified according to criteria described in Levin et al (2009) as a condition with capacity for 'true recovery', e.g. stroke (39\%); a neurodegenerative condition, e.g. Parkinson's disease (19\%); a condition with capacity for 'compensatory recovery', e.g. cerebral palsy $(14 \%)$; and a small percentage were classified as a mobility condition (19\%).
Individuals attending the community rehabilitation service were asked whether they received informal care. If the primary caregiver was present, verbal consent was obtained and the carer was asked to fill in a written survey. They were informed that they could complete it at the time or take it home and complete it later. When carers did not attend the service, questionnaires were given to the individual with a neurological condition to take home to their carer to complete. A reply paid envelope was provided to allow carers to return questionnaires by mail as well as in person.

Those individuals who did not consent did not complete the surveys. Similar to the care recipients, carers completed the questionnaire at baseline and 3-month follow-up. If the carer was not present at the follow up, the care recipient was asked to give the questionnaire to their carer to complete and post back.

The economic impact of caregiving on the carer was measured using an adapted carer questionnaire at baseline and at three-month follow up (Wimo et al, 2002). Three basic components of informal care were examined:

Support with basic activities in daily living (e.g. dressing, hygiene, eating)

Instrumental activities in daily living (e.g. meal preparation, shopping)

Supervision.

Carers were asked to describe the time spent in the various activities each day and week as appropriate. The 3-month questionnaire also included questions regarding the impact of their caring responsibilities on their employment. Details were sought as to whether the carer had changed work status (full-time, part-time, casual, unemployed), number of hours worked per week, and their average range of income.

The utility (or benefit) people gained from caring was measured using the Carer Experience Scale (CES) (Al-Janabi et al, 2011) at baseline and at 3 months. The CES, a preference-based measure, was specifically designed for use in economic evaluation and is scored on a $0-100$ scale. This measure focuses on carer-related quality of life rather than health-related quality of life. The CES incorporates six attributes that people value when providing care to older people. These attributes are:

Getting on (i.e. getting on with the person they are caring for)

Organisational assistance

Social support

Activities

Control

Fulfilment. 
Each of the six attributes has three levels of responses (mostly, sometimes, rarely). The scale has been shown to have construct validity (Goranitis et al, 2014) and was developed using a meta-ethnography process to derive the appropriate domains that were important to carers (Al-Janabi et al, 2008).

\section{Data analysis}

The cost of replacing informal care with formal care was costed using a proxy good method (van den Berg et al, 2006). The hours spent caring were multiplied by the wage rate of a paid community care worker using published award rates (A \$22.82 per hour; Fair Work Ombudsman, 2013). The reduction in work hours for each carer was calculated from self-reported reduction in hours or loss of job. The economic cost of the loss of income from caring was calculated using the average Australian wage rate multiplied by the reduction in work hours from caring and presented in Australian dollars (A\$) where A \$1 €0.71 (exchange rate in 2014).

Utility scores were derived for the CES using UK algorithms (Al-Janabi et al, 2011) as no Australian values have been published. Results of the CES are presented descriptively using the overall utility scores. Pre-post comparison was conducted using a paired t-test.

\section{RESULTS}

Forty-two carers completed the questionnaire at baseline and 30 completed the follow-up at
3 months. Demographics were not collected on the carers. The most common primary diagnosis of the care recipients was stroke $(38 \%)$, multiple sclerosis (19\%) and orthopaedic or mobility issues (19\%). The average age of the person undertaking the rehabilitation programme was 67 years old and $41 \%$ were female. At baseline, those participants whose had carers complete the survey had an average Lawton's score (Lawton and Brody, 1969) of 21.1 compared with 24.1 for all participants of the service, indicating a higher level of dependency. At the three-month follow-up, care recipients had an average Lawton's score of 22.2 compared with 25.4 for all participants at the centre.

Table 1 shows a breakdown of the daily time foregone in providing informal care for someone receiving rehabilitation. Carers spent on average 28 hours per week on various activities relating to caring. Activities most commonly performed and that were identified by caregivers to require the most amount of time a week were preparing meals (average of $\sim 14$ hours a week), transporting the person they care for, and shopping with or for the person they care for. Care time per carer did not reduce pre- and post-rehabilitation. The cost of replacing this care time with a paid community care worker was estimated to be over A $\$ 650$ per person, per week of care. Figure 1 presents the percentage of carers involved in each caring activity. A majority of carers assisted with transporting ( 80\%), shopping $(\sim 70 \%)$ and preparing meals $(\sim 90 \%)$ for the person.

Employment status and income are presented in Table 2. The majority of carers received carer

\begin{tabular}{|l|l|l|}
\hline \begin{tabular}{l} 
Table 1. Average time spent on caring activities \\
\hline Informal carer time
\end{tabular} & $\begin{array}{l}\text { Baseline } \\
\text { Time } \\
\text { Mean (SD) }\end{array}$ & $\begin{array}{l}\text { Three month } \\
\text { Mean (SD) }\end{array}$ \\
\hline Time spent (hours/day) & & \\
\hline Preparing meals & $2.0(1.2)$ & $1.6(1.0)$ \\
\hline Assisting with eating & $0.3(0.1)$ & $0.4(0.1)$ \\
\hline Supervising eating & $1.0(0.6)$ & $0.7(0.3)$ \\
\hline Helping with showering and toileting & $0.6(0.7)$ & $0.8(0.5)$ \\
\hline Helping the person get dressed & $0.3(0.2)$ & $0.4(0.2)$ \\
\hline Other general help and supervision & $1.6(2.0)$ & $1.9(1.5)$ \\
\hline Time spent (hours/week) & & $2.6(2.2)$ \\
\hline Shopping with the person & $2.5(2.1)$ & $2.2(1.3)$ \\
\hline Shopping for the person (on your own) & $1.8(0.8)$ & $3.5(3.2)$ \\
\hline Transporting the person & $3.3(3.7)$ & $1.1(0.9)$ \\
\hline Helping with finances & $1.3(1.0)$ & $28.2(22.6)$ \\
\hline Total amount spent on all activities(hours/week)* & $28.5(20.1)$ & \\
\hline *Column does not add to total as some activities were per day and some per week & & \\
\hline
\end{tabular}




\section{Table 2. Employment and income}

\section{Description}

Receive a carer payment (Yes)

Carer is currently employed (Yes)

Number of hours carer works per week, mean (SD)

Personal income range of the carer

$<\$ 15,000$

$\$ 15,000$ to $\$ 30,000$

$\$ 30,000$ to $\$ 50,000$

$\$ 50,000$ to $\$ 70,000$

$>\$ 70,000$

Changed work status due to caring

Carer has resigned work due to caring responsibilities

Carer has recently refused a promotion due to caring responsibilities

Personal activities

Carer has cut back on personal activities

Extra time/week the carer would prefer to be spending on personal activities

None

$11 / 30(36.7 \%)$

0-5 hours

$13 / 30(43.3 \%)$

$3 / 30(10.0 \%)$

$3 / 30(10.0 \%)$

Number $(\%)$ of carers

affected

$18 / 30(60.0 \%)$

$5 / 29(17.2 \%)$

$34.8(15.3)$

$3 / 22(13.6 \%)$

$9 / 22(40.9 \%)$

$5 / 22(22.7 \%)$

$1 / 22(4.6 \%)$

$4 / 22(18.2 \%)$

$12 / 29(41.4 \%)$

$10 / 27(37.0 \%)$

$1 / 29(3.5 \%)$

$5-10$ hours

$>10$ hours

*Not all variables are out of 30 as data was incomplete on some variables allowance, a non-means tested government allowance of around A \$180 per week (Australian Government, 2014). Most carers were currently unemployed and around $41 \%$ had changed their work status due to caring. Two carers changed their work status from full-time to part-time and 10 of the carers had left employment due to caring responsibilities. The economic loss due to underemployment in these households is substantial. At the average equivalent disposable income in Australia of A $\$ 918$ (Australian Bureau of Statistics, 2013), each full-time job loss costs a household $\mathrm{A} \$ 48,000$ per annum and each reduction in work from full-time to parttime A $\$ 24,000$ per annum. For this cohort of 29 carers, it is estimated that caring responsibilities have cost households A $\$ 324,000$ per year in lost employment.

Of 42 carers interviewed, 10 had complete baseline and follow-up data for the CES and were able to be analysed. An average improvement of around 6 points was recorded from 69 at baseline to 75 at 3 months; however, this improvement was not statistically significant $(P=0.056)$. In terms of carer's quality of life, $70 \%$ of carers reported having to reduce personal activities due to caring, with $64 \%$ identifying they would like to spend at least 5 hours a week engaging in activities outside of caring (Table 2).

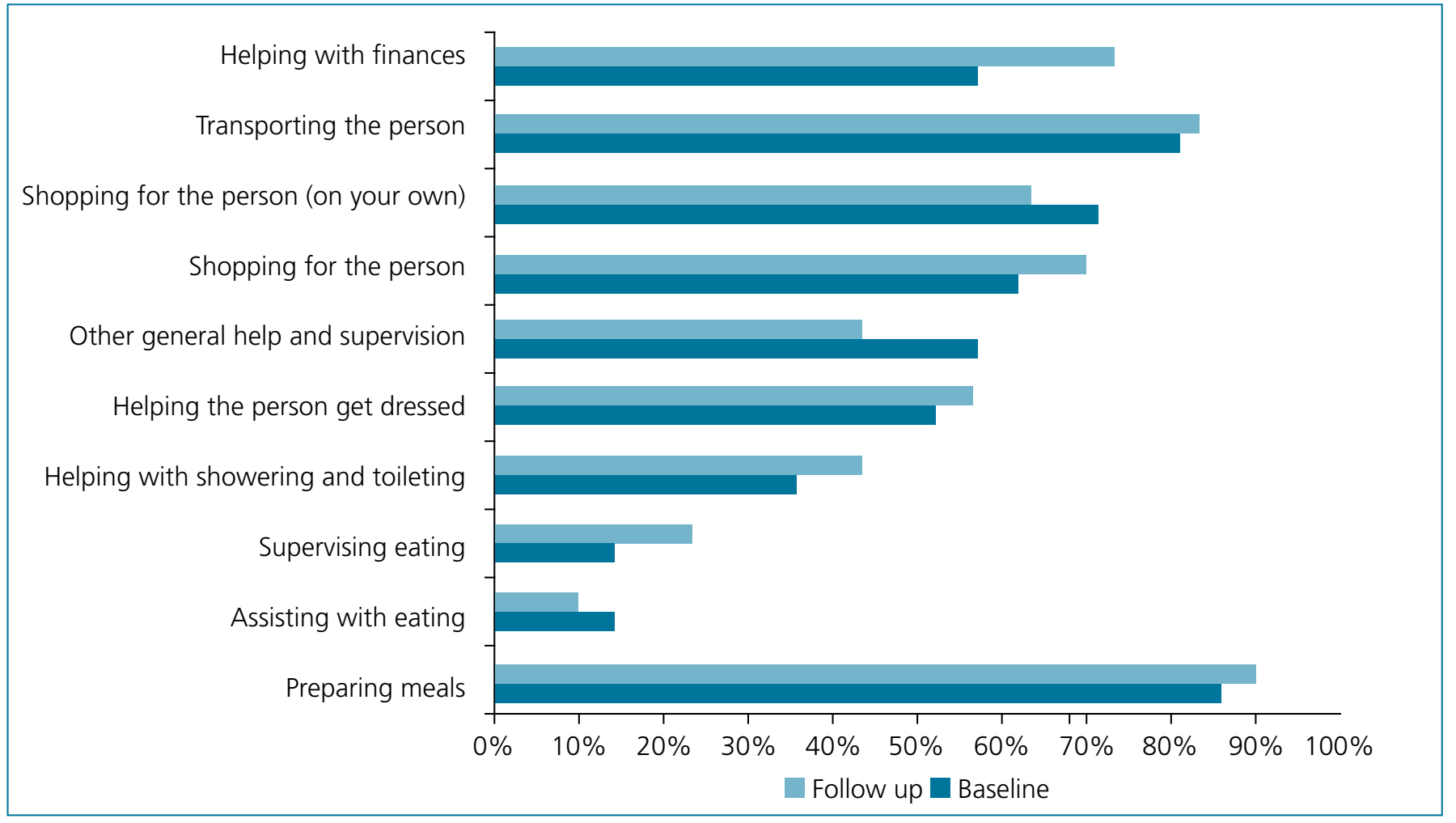

Figure 1. Percentage of carers involved in each caring activity at baseline and 3-month follow up 


\section{DISCUSSION}

The aim of the current study was to explore the economic and quality of life impact of caregiving for those providing care to individuals receiving a community rehabilitation service. The findings indicate that providing care to individuals receiving rehabilitation for neurological conditions impacts substantially on caregivers-financially and personally. We also found that while rehabilitation may improve the functional ability of the care recipient, it does not necessarily reduce the demands for informal care.

While this study focused on people with a neurological condition, the type of care needed to be provided is similar to Australian data on carers from the survey on Disability Ageing and Carers (Australian Bureau of Statistics, 2012) where informal carers assisted with paperwork $(86 \%)$, transport $(82 \%)$, meal preparation $(81 \%)$, self-care $(74 \%)$, and mobility $(71 \%)$.

The unpaid work that carers do represents a significant economic resource. If all carers in Australia ( 500,000 people) spend a similar time each week in caring activities as the cohort in this study, the replacement value of this care would be around A $\$ 16$ billion per year. Other studies have found that the value of informal care is indeed large with an estimated cost of replacing informal care with formal care providers in Australia, of A $\$ 30.5$ billion dollars a year (Access Economics, 2005). An American study estimated the replacement cost at US\$196 billion in 1997, a figure that dwarfs actual spending on formal care (Arno et al, 1999).

This study supports previous research that identified that carers have had to reduce paid work, are more likely to be living in poverty, and have subsequent sequelae of ill health (Hill et al, 2011). The current study found that carers had lost an average of $\mathrm{A} \$ 11,000$ per year in income in order to fulfil their caring duties. This is a conservative estimate, as the current data did not account for loss from not taking up higher positions or better paid jobs. While this may not necessarily be a loss to the economy (as other people take these positions), the loss to the individuals and their families is considerable, and these households are put at risk of financial hardship where paid work is replaced with caring duties.

In a full economic evaluation, particularly for conditions where caring is vital to the wellbeing of the participant, both the participant's and the carer's quality of life should be incorporated into the evaluation. The Carer Experience
Scale (CES) is to our knowledge the only utility scale developed for carers. The current study demonstrated that the CES was a feasible measure to collect and while no significant change was seen, possibly due to the small cohort, improvement in carer experience was observed over a rehabilitation journey. Future investigation into the wellbeing and quality of life impact of caregiving could also explore the psychological, physical and social health of carers in order to determine whether data obtained using the CES is the most appropriate measure of carer experience for those caring for individuals with neurological conditions.

\section{Limitations}

Although a primary goal of rehabilitation is to increase the person's functional capacity and therefore reduce the need for carer assistance, we did not find any reduction in carer hours post rehabilitation. However, this study was potentially limited in that follow-up was only carried out once, and only after 3 months. During this time, carers may have had higher requirements, particularly for transport as people were undergoing active rehabilitation. Future study should take a longitudinal approach and follow-up over an extended period of time (at least 12 months) to more adequately capture the rehabilitation process and impact to carers post rehabilitation. Such an approach would more adequately identify the impact of rehabilitation on a caregiver's economic and quality of life status.

Another potential issue to be addressed is that the rehabilitation targets the person and not necessarily the person/carer interaction. Roles may be set in place and habits may prevent change from occurring. There may need to be active carer intervention to enable role change and allow the person to take on more of their own care in areas such as food preparation and dressing.

The present study is predominantly limited by the small sample of caregivers that responded. Carers were only recruited if they attended the appointment with the client and therefore only a limited number were available. Carers were sampled alongside a service study of a community rehabilitation service and the study was not designed specifically to ensure an adequate recruitment of carers. Nevertheless, most studies of carers focus on the psychological impacts of caring (strain and stress). In this study we have shown that substantial economic costs are also personally accrued by carers and their families. Economic impact extends to the 
carer's utilisation of health resources (Peters et al, 2013), which was not captured in this study. Future investigation could also identify whether carers are accessing health services themselves and whether the rehabilitation of the person they are caring for, lessens the need for them to access such services. The strength of this study, however, was that individual data was collected on different activities of caring in a cohort with neurological conditions and the impact of caring on employment status was measured.

\section{CONCLUSIONS}

Informal carers are an essential part of the care of people with neurological conditions. This study in a community rehabilitation setting shows that there is a considerable, measurable, financial impact of caring responsibilities on the carer and their household. These impacts along with the utility derived by carers should be incorporated into economic evaluations of rehabilitation programmes in order to assess the true costs and benefits of these programmes. IJTR

\section{Conflict of interest: none declared.}

Access Economics (2005) The Economic Value of Informal Care. Access Economics, Canberra

Al-Janabi H, Coast J, Flynn TN (2008) What do people value when they provide unpaid care for an older person? A metaethnography with interview follow-up. Soc Sci Med 67(1): 111-21. doi: 10.1016/j.socscimed.2008.03.032

Al-Janabi H, Flynn TN, Coast J (2011) Estimation of a preference-based carer experience scale. Med Decis Making 31(3): 458-468. doi: 10.1177/0272989X10381280

Arno PS, Levine C, Memmott MM (1999) The economic value of informal caregiving. Health Aff 18(2): 182-8. doi: 10.1377/hlthaff.18.2.182

Australian Bureau Of Statistics (2012) Disability, Ageing and Carers, Australia: Summary of Findings. http://bit. ly/2dzxiOR (accessed 22 September 2016)

Australian Bureau Of Statistics (2013) Household Income and Income Distribution, Australia, 2011-12. http://bit.
ly/2cKWWFr(accessed 22 September 2016)

Australian Government (2014) Carer Allowance. https:// www.humanservices.gov.au/customer/services/centrelink/ carer-allowance (accessed 22 September 2016)

Blake H, Lincoln NB, Clarke DD (2003) Caregiver strain in spouses of stroke patients. Clin Rehabil 17(3): 312-7. doi: $10.1191 / 0269215503 \mathrm{cr} 613 \mathrm{oa}$

Chipchase SY, Lincoln NB (2001) Factors associated with carer strain in carers of people with multiple sclerosis. Disabil Rehabil 23(17): 768-76.

Fair Work Ombudsman (2013) Social, Community, Home Care and Disability Services Industry Award 2010 [MA000100]. Pay and Conditions Guide. Australian Government, Canberra

Goranitis I, Coast J, Al-Janabi H (2014) An investigation into the construct validity of the Carer Experience Scale (CES). Qual Life Res 23(6): 1743-52. doi: 10.1007/s11136-0130616-1

Gunnell D, Coast J, Richards SH, Peters TJ, Pounsford JC, Darlow MA (2000) How great a burden does early discharge to hospital-at-home impose on carers? A rand omized controlled trial. Age Ageing 29(2): 137-42. doi 10.1093/ageing/29.2.137

Hughes SL, Giobbie-Hurder A, Weaver FM, Kubal JD, Henderson W (1999) Relationship between caregiver burden and health-related quality of life. Gerontologist 39(5): 534-45. doi: 10.1093/geront/39.5.534

Iecovich E (2008) Caregiving Burden, Community services, and quality of life of primary caregivers of frail elderly persons. J Appl Gerontol 27(3): 309-30. doi 10.1177/0733464808315289

Lawton, MP, Brody EM (1969) Assessment of older people: self-maintaining and instrumental activities of daily living. Gerontologist 9(3): 179-86.

Levin MF, Kleim JA, Wolf SL (2009) What do motor "recovery" and "compensation" mean in patients following stroke? Neurorehabil Neural Repair 23(4): 313-9. doi: $10.1177 / 1545968308328727$

Low JT, Payne S, Roderick P (1999) The impact of stroke on informal carers: a literature review. Soc Sci Med 49 711-25. doi: 10.1016/S0277-9536(99)00194-X

Peters M, Jenkinson C, Doll H, Playford ED, Fitzpatrick $\mathrm{R}$ (2013) Carer quality of life and experiences of health services: a cross-sectional survey across three neurological conditions. Health Oual Life Outcomes 11(1): 103. doi: 10.1186/1477-7525-11-103

Hill T, Thomson C, Cass B (2011) The Costs of Caring and the Living Standards of Carers. Australian Government, Canberra

van den Berg B, Brouwer W, Van Exel J, Koopmanschap M, Van Den Bos GA, Rutten F (2006) Economic valuation of informal care: lessons from the application of the opportunity costs and proxy good methods. Soc Sci Med 62(4): 835-45. doi: 10.1016/j.socscimed.2005.06.046

Wimo A, Von Strauss E, Nordberg G, Sassi F Johansson L (2002) Time spent on informal and formal care giving for persons with dementia in Sweden. Health Policy 61(3): 255-68. doi: 10.1016/S0168-8510(02)00010-6

\section{Support and care for patients with long-term conditions}

\section{Helen McVeigh}

Each chapter presents learning points, using a reflective approach

Essential guide to long-term conditions, exploring the key principles of practice, skills and policy

- Case history examples included to illustrate issues discussed

ISBN-13: 978-1-85642-392-2; 234 x 156 mm; paperback; 280 pages; publication 2010; £24.99

\section{Order your copies by visiting} www.quaybooks.co.uk

\author{
or call our Hotline \\ $+44(0) 1722716935$
}

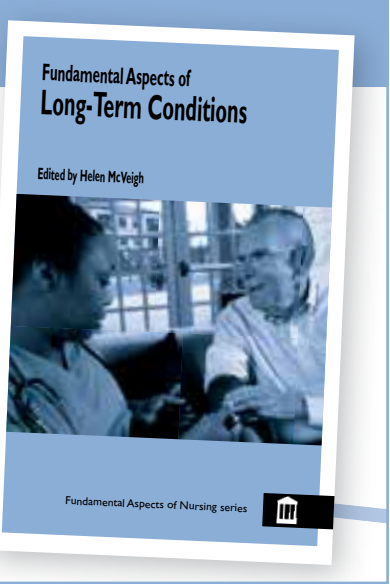

